\title{
Cosmological magnetic field seeds produced by the Weibel instabilities
}

\author{
M. Lazar*, R. Schlickeiser and T. Skoda \\ Institut für Theoretische Physik IV, Ruhr-Universität Bochum, D-44780 Bochum, Germany \\ *email: mlazar@tp4.rub.de
}

\begin{abstract}
The source of the cosmological magnetic field is still unknown because the widely invoked dynamo processes are only able to regenerate and amplify some initial magnetic field seeds. In the hot and highly ionized intergalactic matter such magnetic field seeds can easily be produced by the (electro-)magnetic instabilities of Weibel type. Here we discuss suplementary mechanisms that can make these Weibel created fields to evolve at large scales presently observed in galaxies and clusters and can also enhance these magnetic field seeds after the dissipation.
\end{abstract}

Keywords. cosmology: large-scale structure of universe, plasmas, instabilities, magnetic fields

\section{Introduction}

Dynamo mechanism presently widely invoked in cosmological magnetic field genesis need some seeds to work with. Here we show that such seed fields can originate from the Weibel instabilities (Weibel 1959). These instabilities are driven by various kinetic anisotropies, e.g., heating flows, shocks and interpenetrating plasma shells, and generate long-lived quasistatic magnetic fields providing plausible explanations for the origin of cosmological magnetic field (Schlickeiser \& Shukla 2003) or the magnetic boosts in astrophysical sources (GRBs, SNRs) of nonthermal radiation (Medvedev \& Loeb 1999).

\section{Weibel instabilities}

The instabilities of Weibel-type operate in initially unmagnetized plasma systems: the filamentation instability driven by a relative motion of different plasma shells (beamplasma, counterstreams), Weibel instability driven by a thermal anisotropy, or the cumulative Weibel/filamentation instability driven by counterstreaming plasmas with intrinsic kinetic anisotropies. Any magnetic pertubation generates currents of charged particles, which reinforce the perturbation (Ampere's law) to grow to large amplitudes. It saturates due to the magnetic trapping of plasma particles, when the magnetic bounce frequency $\left(\Omega^{2} \equiv e k_{\max } v B_{\max } /(m c)\right)$ increases to a value comparable to the linear growth rate prior to the saturation $\Omega_{\max } \simeq \Gamma_{\max }$ (Davidson 1972). The mode with the largest growth rate, $\Gamma_{\max }\left(k_{\max }\right)$, dominates and sets the characteristic length scale of the magnetic field fluctuations, $\lambda \sim k_{\text {max }}^{-1}$ at the saturation. Particles free streaming across the excited magnetic field lines is suppressed once the particle's gyroradius $\rho=v / \Omega_{\max }=e B_{\max } /(\mathrm{mc})$ becomes comparable to the length scale of the magnetic field fluctuations, $k_{\max }^{-1}$, yielding (Medvedev \& Loeb 1999) for the maximum $B_{\max } \simeq m v c k_{\max } / e=m c \Gamma_{\max } / e$.

Saturated magnetic field have been derived for the filamentation instability $B_{\max }=$ $v_{0} \sqrt{4 \pi n m / \gamma_{0}}$, the Weibel instability $\left(v_{t h}=v_{t h, 2}>>v_{t h, 1}\right) B_{\max }=\sqrt{2 \pi n m} v_{t h}$ and for the cumulative Weibel/filamentation instability $B_{\text {max }}=\sqrt{4 \pi n m\left(v_{t h}^{2}+2 v_{0}^{2}\right)}$, and these magnetic fields are consistent with the observations: $B=\eta B_{\max } \sim 10^{-8}-10^{-6} \mathrm{G}$ in 
clusters, $\sim 10^{-7}-10^{-5} \mathrm{G}$ in intergalactic medium, $\sim 10^{-7} \mathrm{G}$ in galaxies, for a conversion factor $\eta=0.01-0.1$ (Schlickeiser \& Shukla 2003, Lazar et al. 2009).

By comparison to the cosmological magnetic field presently measured on scales of galaxies or clusters $(\gtrsim 1 \mathrm{kpc})$, the coherence length scales of the Weibel created fields (of the order of plasma skin depth, $c / \omega_{p p}<10^{5} \mathrm{~km}$ ), are very small.

\section{Evolution of the Weibel magnetic field to large cosmological scales}

The Weibel fields do not decay much after the saturation (Silva et al. 2003), and these fields may become coherent up to very large scales expected to reach kpc scales through inverse cascade processes (Cho \& Vishniac 2000). Inverse cascades produce in plasma turbulence, and trasfer energy towards large scales. In a weak-turbulence model, inverse cascades are described by the theory of nonlinear wave interactions. For the Weibel field, the main channels of resonant wave-wave conversion after the saturation were identified according to long-time evolution in numerical experiments, indicating an inverse cascade with small-scale structures merging into large scale coherent fields (Lazar et al. 2010).

For a long term evolution to large cosmological time and space scales these magnetic fields dissipates by many order of magnitudes. However, recent numerical experiments have shown that the weak seeds are again enhanced by the turbulent flow motions (Ryu et al. 2008). Intense fields are obtained at large coherence scales of the order of dominant eddies (curvature radius of typical cosmological shocks). Thus, the turbulence excited during the process of large-scale structure formation can amplify very weak strengths of the intergalactic magnetic field (after the dissipation), e.g., $B \geqslant 10^{-10} \mathrm{G}$, and reach coherence length $\sim 100 \mathrm{kpc}$, fitting well the presently observed magnetic fields.

Amplification of the galactic magnetic field seeds can be attributed to turbulent flows and dynamos observed and predicted in convective zones of the stars and planets, stellar wind and supernova explosions, star forming regions, interstellar medium (Beck 2007), or by the ( $\alpha-\Omega$, mean field) dynamos in stars (the Sun), accretion disks (protostellar disks, close binaries, AGNs). Intergalactic magnetic field can be enhanced by turbulent dynamo in accretion disks and motion of galaxies through IGM, or by $(\alpha-\Omega$, mean field) dynamos in spiral galaxies, giant molecular clouds (Ryu et al.2008).

\section{Conclusions}

The instabilities of Weibel-type can potentially create magnetic seed fields and their evolution to large cosmological scales is sustained by the amplification mechanisms and the inverse cascades in plasma turbulence predicted in galaxies and intergalactic medium.

\section{References}

Beck, R. 2007 A\&A, 470, 539

Schlickeiser, R. \& Shukla, P. K. 2003, ApJ (Letters), 599, L57

Lazar, M., Schlickeiser, R., Wielebinski, R., \& Poedts, S. 2009, ApJ, 693, 1133

Weibel, E. S. 1959, Phys.Rev.Lett., 2, 83

Davidson, R. C., Hammer, D. A., Haber, I., \& Wagner, C. E. 1972, Phys.Fluids, 15, 317

Medvedev, M. \& Loeb, A. 1999, ApJ, 526, 697

Ryu, D., Kang, H., Cho, J. \& Das, S. 2008, Science, 320, 909

Silva, L. O., Fonseca, R. A., Tonge, J. W., Dawson, J. M., Mori, W. B., \& Medvedev, M. V. 2003, ApJ (Letters), 596, L121

Cho, J. \& Vishniac, E. T. 2000, ApJ, 539, 237

Lazar, M., Lapenta, G., Schlickeiser, R., \& Poedts, S. 2010, Phys. Plasmas, in preparation 